\title{
A ABCS Health Sciences agradece a valiosa colaboração de seus revisores científicos em 2014
}

DOI: http://dx.doi.org/10.7322/abcshs.v39i3.658

Adriana da Silva Santos - IFG - Goiânia/GO

Adriana Silveira de Almeida - UFRGS - Porto Alegre/RS

Afranio Donato de Freitas - Hospital Ortopédico - Belo Horizonte/MG Alessandra Bento Veggi David - FIOCRUZ - Rio de Janeiro/RJ Alessandra Silva Dias de Oliveira - UFRJ - Rio de Janeiro/RJ Alexandre Vianna Montagnero - UFU - Uberlândia/MG Ana Lúcia de Mattia - UFMG - Belo Horizonte/MG Anderson Ravy Stolf - UFRGS - Porto Alegre/RS Andrea de Andrade Ruggiero - FMABC - Santo André/SP Anelise Impelizieri Nogueira - UFMG - Belo Horizonte/SP Anselmo José Perez - UFES - Vitória/ES Bruno da Cruz Pádua - CEFET - Belo Horizonte/MG Caio Mauricio Mendes de Cordova - FURB - Blumenau/SC Camila Isabel Santos Schivinski - UDESC - Florianópolis/SC Carin Weirich Gallon - UCS - Caxias do Sul/RS Carlos Bandeira de Mello Monteiro - USP - São Paulo/SP Cássia Cinara da Costa - FEEVALE - Novo Hamburgo/RS Celiane Camargo-Borges - NHTV - Breda/Holanda Cristiane Paulin Simon - UFTM - Uberaba-MG Daniel Fraga - UFMS - Coxim/MS

Daniela de Oliveira Melo - UNINOVE - São Paulo/SP

Dartagnan Pinto Guedes - UNOPAR - Londrina/PR

Edio Luiz Petroski - UFSC - Florianópolis/SC

Elaine Drehmer de Almeida Cruz - UFPR - Curitiba/PR

Eli Maria Pazzianotto Forti - UNIMEP - Piracicaba/SP

Eliana Amaral - UNICAMP - Campinas/SP

Fabiana Schuelter-Trevisol - Unisul - Tubarão/SC

Fabrício Luiz Assini - FESSC - São José/SC

Flávio Tarasoutchi - USP - São Paulo/SP

Flora Magno de Jesus Oliveira - FIOCRUZ - Manguinhos/RJ Francisca Maria Assmann Wichmann - UNISC - Santa Cruz do Sul/RS Gabriela Arantes Wagner - USP - São Paulo/SP

Giane Moliari Amaral Serra - UNIRIO - Rio de Janeiro/RJ

Gibran Roder Feguri - UFMT - Cuiabá/MT
Gilson de Vasconcelos Torres - UFRN - Natal/RN

Irami Araujo Filho - UFRN - Natal/RN

Isabelle Katherinne Fernandes Costa - UFRN - Natal/RN Jair Rodrigues Garcia Júnior - UNOESTE -Presidente Prudente/SP Janaína Vieira dos Santos - UFPEL - Pelotas/RS Janaísa Gomes Dias de Oliveira - PUC - Porto Alegre/RS Joaquim Carlos Rossini - UFU - Uberlândia/MG José Antonio Chehuen Neto - UFJF - Juiz de Fora/MG Larissa Chaves Pedreira - UFBA - Salvador/BA Liana Barbaresco Gomide - UnB - Brasília/DF Livia Carla de Melo Rodrigues - UFES - Vitória/ES Lorita Marlena Freitag Pagliuca - UFC - Fortaleza/CE Lourdes Conceição Martins - UNISANTOS - Santos/SP Lucia Caruso - USP - São Paulo/SP Luciane Aparecida Pascucci Sande de Souza - UFTM - Uberaba/MG Luciano de Figueiredo Borges - UFMG - Belo Horizonte/MG Lucio Garcia de Oliveira - USP - São Paulo/SP Luís Carlos Carvalho da Graça - IPVC - Viana do Castelo/Portugal Marco Antonio Marcolin - USP - São Paulo/SP Marcus Vinicius João Luis - USP - São Paulo/SP Maria Alayde Mendonça da Silva - UFAL - Maceió/AL Maria Alves Barbosa - UFG - Goiânia/GO Maria Beatriz Lisboa Guimarães - UFPE- Recife/PE Maria Cristina Pinto de Jesus - UFJF - Juiz de Fora/MG Maria do Carmo Andrade Duarte de Farias - UFCG - Cajazeiras/PB Maria Inês de Toledo - UnB - Brasília/DF Maria Ribeiro Lacerda - UFPR - Curitiba/PR Mario Oliveira Lima - UNIVAP - São José dos Campos/SP Marta Fioravanti Carpes - UNIPAMPA - Uruguaiana/RS Mathias Roberto Loch - UEL - Londrina/PR Mauro Henrique Nogueira Guimarães de Abreu - UFMG - Belo Horizonte/MG Melissa Medeiros Braz - UFSM - Santa Maria/RS Michelle Delboni dos Passos - UERJ - Rio de Janeiro/RJ Michelli Cristina Silva de Assis - UFRGS - Porto Alegre/RS 
Miguel Angelo Alves dos Santos - UVV - Vila Velha/ES Milton Luiz Gorzoni - FCMSCSP - São Paulo/SP

Nelci Terezinha Zorzi - UPF - Passo Fundo/RS

Odilon Salim Costa Abrahin - UEPA - Belém/PA

Patrícia Lima Ventura - UESPI - Teresina/PI

Paula Paraguassú Brandão - CUCL - Rio de Janeiro/RJ

Pedro Pereira Tenório - UFPE - Caruaru/PE

Pedro Shiozawa - FCMSCSP - São Paulo/SP

Rafaello Pinheiro Mazzoccante - UCB - Brasília/DF

Raphael Mendes Ritti Dias - UPE - Recife/PE

Reginaldo Teixeira Mendonça - UFG - Goiânia/GO

Ricardo Kaempf de Oliveira - UFPEL - Porto Alegre/RS

Ricardo Pedrozo Saldanha - Unilasalle - Canoas/RS

Rita Batista Santos - UFRJ - Rio de Janeiro/RJ

Rodrigo Daminello Raimundo - UniFMU - São Paulo/SP

Rodrigo Leal Alves - UFBA - Salvador/BA
Rodrigo Pinheiro de Toledo Vianna - UFPB - João Pessoa/PB

Ruy Laurenti - USP - São Paulo/SP

Sara Regina Meira Almeida - UNICAMP - Campinas/SP

Silmara Aparecida de Oliveira Leite - UP - Curitiba/PR

Sofia José Nunes Nóbrega - UL - Lisboa/Portugal

Sonia Regina Pasian - USP - Ribeirão Preto/SP

Stella Maris Hildebrand - UnB - Brasília/DF

Susanne Elero Betiolli - UFPR - Curitiba/PR

Táki Athanássios Cordás - USP - São Paulo/SP

Talita Fortunato-Tavares - USP - São Paulo/SP

Tania Cristina Santos Andrade - UniCEUB - Brasília/DF

Valter Cordeiro Barbosa Filho - UFSC - Florianópolis/SC

Vandré Casagrande Figueiredo - Univ. Auckland - Auckland/Nova Zelândia

Viviane Euzébia Pereira Santos - UFRN - Natal/RN

Viviani Barnabé - Harvard University - Boston/EUA 